\title{
Inducing Chaos in MOSFET-Based Electronic Circuits
}

\author{
Srinivasan Gopal · Ying-Cheng Lai
}

Received: 1 September 2007 / Revised: 5 June 2008 / Published online: 3 April 2009

(C) Birkhäuser Boston 2009

\begin{abstract}
We construct a nonlinear, MOSFET-based electronic circuit and address the question of inducing chaos. A recently proposed method makes use of resonant perturbations, which is applicable to situations where chaos is desired, under the following three constraints: (1) the circuit operates in a stable periodic regime, (2) no parameters or state variables of the circuit are directly accessible to adjustment, and (3) the circuit equations are not available. We argue that phase-locked loops can be utilized to realize resonant perturbations in terms of frequency and phase match, and demonstrate experimentally that chaos can be induced in the MOSFET circuit.
\end{abstract}

Keywords Low-power circuits · MOSFET · Chaos · Lyapunov exponent · Phase-locked loops

\section{Introduction}

Inducing and maintaining chaos has been an active research area in nonlinear dynamics. For example, there are biological situations where chaotic behavior is desirable [15], and previous works have demonstrated that chaos can be induced by applying small perturbations to an available parameter or dynamical variable of the system $[6,9,15]$. However, in existing works on inducing or maintaining chaos, either the system is in a transiently chaotic regime with some externally accessible parame-

S. Gopal · Y.-C. Lai $(\bowtie)$

Department of Electrical Engineering, Arizona State University, Tempe, AZ 85287, USA

e-mail: Ying-Cheng.Lai@asu.edu

S. Gopal

e-mail: Srinivasan.Gopal@asu.edu

Y.-C. Lai

Department of Physics, Arizona State University, Tempe, AZ 85287, USA 
ter or state variable, or the system equations are available $[1-4,6,7,9,11-13,15,16$, 20]. For instance, in the experimental work on chaos maintenance by In et al. [6], it is required that the system exhibit intermittency between chaotic and periodic phases, i.e., there is transient chaos and, in addition, an accessible system parameter or variable be available. In another general theoretical work on chaos maintenance using weak harmonic perturbations [3], both system equations and transient chaos are required.

It may also be desirable to induce chaos in nonlinear electronic circuits operating in a stable regime. Examples are circuits embedded in electronic tracking and guidance systems. Chaos may confuse these systems so that it is more likely for them to fail in their intended mission. Inducing chaos to interrupt the normal operation of the circuit can be regarded as advantageous because the absorbed energy required may be much less than that needed to simply "overpower" the same electronics by using large-amplitude excitations. For this type of application, the following three constraints exist: (1) the electronic circuit operates in stable state, (2) the internal structure and parameters of the circuit cannot be modified, i.e., no parameters or state variables of the circuit are directly accessible to adjustment, and (3) the system equations are unknown and only a measured signal from the system may be available. To induce chaos in an electronic circuit under these constraints, a viable way is to subject the circuit to suitably designed external perturbations, such as microwave radiation with special waveforms.

Recently, we have developed a general approach $[8,10]$ to inducing chaos in nonlinear electronic circuits by making use of the principle of phase-locking and resonance, without knowledge of system equations. The idea is to apply judiciously chosen perturbations to drive a target circuit into higher and higher resonant states. The perturbations can be a sinusoidal signal with time-varying frequency and phase, and how they vary is determined by a real-time measured signal from the circuit. The goal is to control the perturbing field so as to maximize its effect on the circuit, thereby driving it as far away from its equilibrium as possible, eventually generating chaos.

The starting point of our theoretical consideration was a simple class of onedegree-of-freedom Hamiltonian systems which, without external perturbations, are fundamentally incapable of exhibiting any chaotic behavior. We argued that smallamplitude, sinusoidal excitations whose frequency and phase match approximately those of the measured signal from the system can drive it gradually into higher and higher energetic states and eventually into chaos. The key role played by the resonant perturbation is then to continuously feed energy into the system to induce instability. We addressed the issue of the time required to induce chaos and obtained a simple scaling relation between this time and the amplitude of the resonant sinusoidal excitation. We then considered the effect of dissipation and demonstrated that, insofar as the average rate of increase of energy in the system is positive, resonant perturbations can drive the system into chaos, whereas the time required satisfies the same scaling law as in the case of Hamiltonian systems. For experimental implementation, we used a prototype of nonlinear electronic circuit, the Duffing circuit, and showed that resonant perturbations from a phase-locked loop (PLL) circuit can readily drive the target circuit into chaos. Here, the role of the PLL circuit is to continuously track the instantaneous frequency and the phase of the target circuit and, at the same time, to deliver 
proper resonant perturbations. We demonstrated that excitation of amplitude from the PLL of about $10 \%$ of the maximum circuit voltage oscillation can induce robust chaotic motion in the circuit. To demonstrate the power of the resonant-perturbation approach, we also showed that in the same parameter regime, random perturbations of much larger amplitude are unable to induce chaos.

In this paper, motivated by the widespread use of metal oxide semiconductor field effect transistors (MOSFETs) in all kinds of electronic devices, we consider MOSFET-based electronic circuits and demonstrate that the principle of resonant perturbation can be applied to induce chaos. First we will construct such a circuit using the current-mode technique, which by itself exhibits regular oscillations. Next, to realize resonant perturbations, we consider a PLL circuit that detects the instantaneous frequency and phase of the voltage oscillations from the MOSFET circuit and delivers a frequency and phase-matched control signal to the circuit. We demonstrate that such resonant perturbations can indeed induce chaos. The induced chaotic behavior is quantified by the largest Lyapunov exponent calculated from a measured voltage signal from the circuit.

Section 2 details the design of our MOSFET-based circuit and experimental setup. Inducing chaos using the scheme of PLL is described in Sect. 3. A discussion is presented in Sect. 4.

\section{Design of MOSFET-Based Nonlinear Circuit}

In our recent work on inducing chaos, the test circuit is one due to Young and Silva [21] that implements the classical Duffing's oscillator in nonlinear dynamics. The standard cubic nonlinear term in the Duffing's equation is replaced by a piecewise linear function, which can be implemented relatively easily. The circuit uses square-wave driving and is capable of chaos-based information processing in a wide frequency range ( $\mathrm{kHz}$ to $\mathrm{GHz}$ ). It has been demonstrated that the Young/Silva circuit preserves the essential dynamics of the original Duffing oscillator, including various periodic and chaotic behaviors [21].

Our experimental circuit here consists of MOS transistors and capacitors [14], which require a relatively low supply voltage $( \pm 1.7 \mathrm{~V})$. The circuit can thus be regarded as a low-power oscillator. The circuit design does not require implementations of mathematical operations such as multiplication. Nonlinearity is embedded in the basic MOS component. The circuit is also scalable over different frequency ranges. The basic block of the circuit is a transconductance-amplifying unit, or a $G_{m}$-cell, which is made up of two MOS transistors (one NMOS and another PMOS). There is no need to use a high-impedance output stage. As a result, incorporating a simple capacitor allows the cell to operate at high frequencies. For this basic cell, the transconductance is given by $G_{m} \approx 2 \beta\left(V_{D D}-V_{T}\right)$, where $\pm V_{D D}$ are the supply voltages, $V_{T n} \approx V_{T p} \equiv V_{T}$ are the threshold voltages, and $\beta$ is a parameter.

The nonlinear oscillator consists of three cascaded $G_{m}-C$ integrators, a current mirror, and a voltage comparator, as shown in Fig. 1 . At node $V_{z}$, we have

$$
I_{1}=-I_{2}+I_{3}-I_{4}+I_{c},
$$


Fig. 1 Block diagram of our nonlinear MOS oscillator made up of three $G_{m}-C$ circuit units

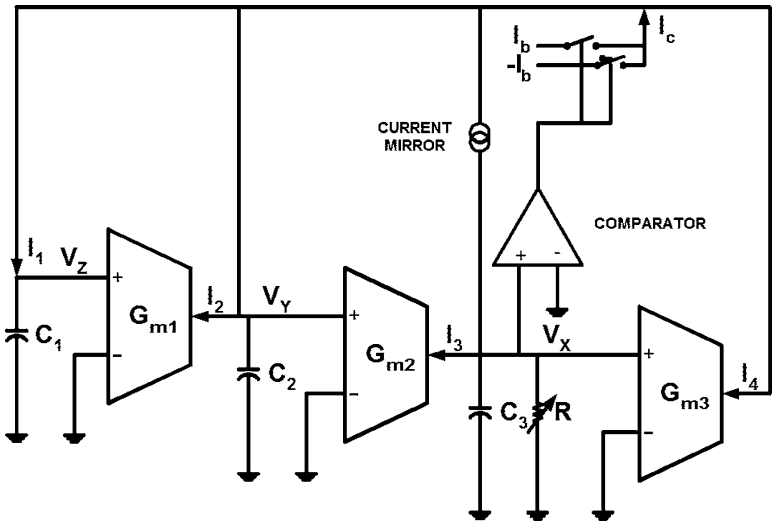

where $I_{2}=G_{m 1} V_{Z}, I_{3}=G_{m 2} V_{Y}$, and $I_{4}=G_{m 3} V_{X}$. The current $I_{c}$ is given by

$$
I_{c}= \begin{cases}I_{b}, & V_{X} \geq 0, \\ -I_{b}, & V_{X}<0,\end{cases}
$$

where $I_{b}$ is the bias current. A basic circuit analysis gives $I_{1}=C_{1} d V_{Z} / d t, I_{2}=$ $-C_{2} d V_{Y} / d t$ and $I_{3}=-C_{3} d V_{X} / d t-V_{X} / R$. We thus have

$$
I_{2}=\frac{C_{2}}{G_{m 2}}\left(C_{3} \frac{d^{2} V_{X}}{d t^{2}}+\frac{1}{R} \frac{d V_{X}}{d t}\right) .
$$

For simplicity, we set $G_{m 1}=G_{m 2}=G_{m 3} \equiv G, C_{2}=C_{3} \equiv C$, and $C_{1} \equiv C / a$. Substituting all the current expressions in (1), we obtain

$$
\begin{aligned}
\frac{d^{3} V_{X}}{d t^{3}}= & -\left(\frac{a G}{C^{2}}+\frac{1}{R C}\right) \frac{d^{2} V_{X}}{d t^{2}}-\left(\frac{a G}{C^{2} R}+\frac{a G^{2}}{C^{2}}\right) \frac{d V_{X}}{d t} \\
& -\left(\frac{a G^{2}}{C^{3} R}+\frac{a G^{3}}{C^{3}}\right) V_{X}-\frac{a G^{2}}{C^{2}} I_{b} f\left(V_{X}\right),
\end{aligned}
$$

where $f\left(V_{X}\right)=1$ for $V_{X} \geq 0$ and $f\left(V_{X}\right)=-1$ for $V_{X}<0$. Note that, while we have derived (3), this is not necessary for our scheme to induce chaos.

Actual implementation of the circuit is shown in Fig. 2, where the basic $G_{m}$ cells are realized by MOSFETs. The value $R$ of the potentiometer can be regarded as a bifurcation parameter. Choosing different values of $R$ can lead to different types of oscillations, e.g., periodic and chaotic. In our experiment, we use N-channel MOSFETs (BS170-ND) and P-channel MOSFETs (ZVP2106A-ND) for the $G_{m}$ cell. The threshold voltages are $V_{T n} \approx V_{T p} \approx 0.6 \mathrm{~V}$. Current mirroring is achieved through transistors $M_{4}, M_{5}, M_{16}$ and $M_{17}$. A simple inverter consisting of $M_{8}$ and $M_{13}$ is used as a voltage comparator, taking advantage of the high gain in the transition region of the inverter. Transistors $M_{10}$ and $M_{11}$ operate as switches, depending on the output of the comparator. The power-supply voltage is $V_{D 0}=1.7 \mathrm{~V}$. The capacitances are $C_{1}=10 \mathrm{nF}$ and $C_{2}=C_{3}=6.6 \mathrm{nF}$. The dynamical variables of the oscillator are $V_{X}$, $V_{Y}$, and $V_{Z}$, which are the voltages across capacitors $C_{3}, C_{2}$, and $C_{1}$, respectively. 


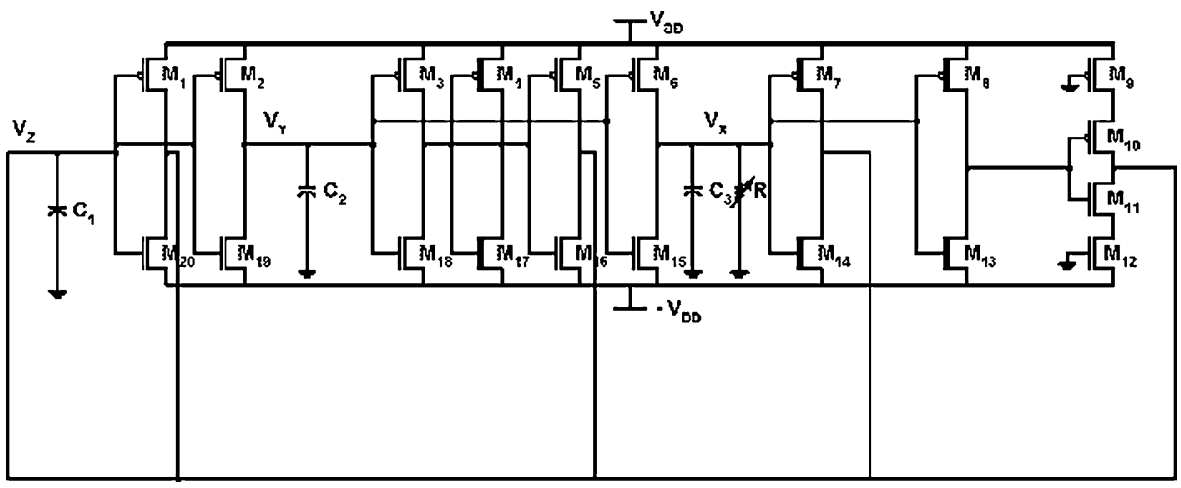

Fig. 2 Actual implementation of circuit in Fig. 1 with MOS transistors

Fig. 3 Experimental setup for inducing chaos by resonant perturbations. A MOSFET oscillator is assumed to be in a stable state initially. A PLL circuit detects a signal from the circuit, say $V_{Y}(t)$, and delivers a small control signal with matched frequency and phase to the oscillator. The outputs of the oscillator are $V_{X}(t)$ and $V_{Z}(t)$, which are recorded by a

standard data acquisition device

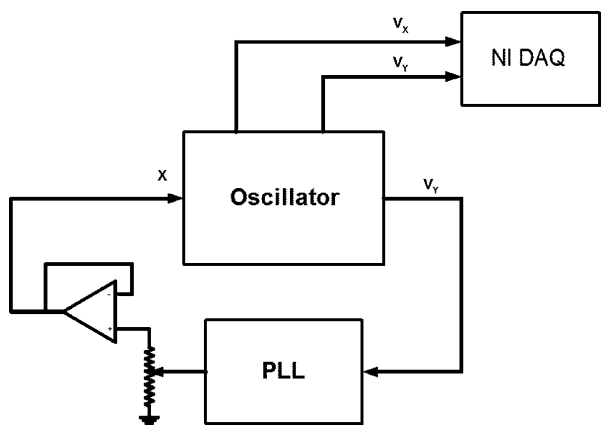

\section{Inducing Chaos by Resonant Perturbations}

\subsection{Use of PLL to Generate Resonant Perturbations}

Our scheme of inducing chaos is shown in Fig. 3, where the PLL circuitry provides a time-dependent, resonant perturbation to a "target" MOSFET circuit. The necessity to use this type of perturbation can be argued qualitatively, as follows. Imagine a simple linear harmonic oscillator. The natural oscillating frequency is an intrinsic parameter, and it does not depend on the energy of the oscillator, a general property of linear oscillators. As a result, one can induce an arbitrarily large disturbance in this system by applying an arbitrarily small perturbing force at the fixed resonant frequency. For a nonlinear system, its natural frequency depends on the energy and is therefore time dependent. In this case, using a perturbing field with a fixed frequency cannot generate resonance. It is then necessary to change the frequency of the perturbing field so as to "follow" the natural frequency of the system as the energy changes due to perturbation $[8,10]$. Another requirement lies in the instantaneous phase of the oscillator. In order to generate chaos, it is necessary to deliver energy into the system so that, in terms of nonlinear dynamics, the system can approach a hyperbolic orbit about which homoclinic and heteroclinic intersections occur [5]. The phase $\phi$ should then be adjusted so that the forcing term is in phase with the system's oscillation. 


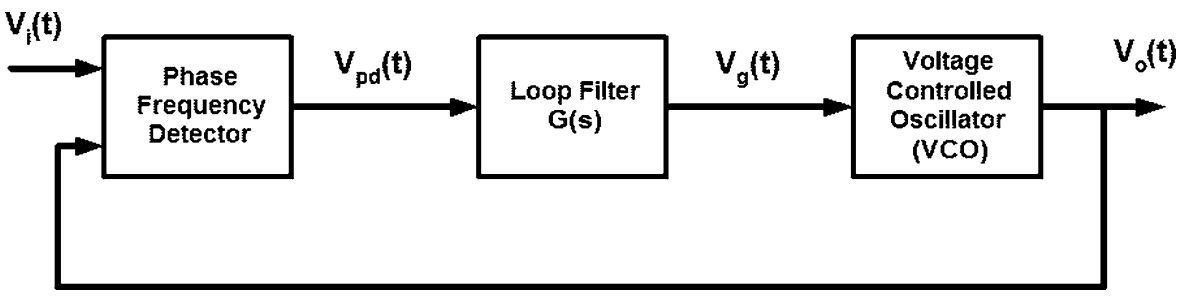

Fig. 4 Block diagram of a PLL. The output signal $V_{0}(t)$ matches the input signal $V_{\mathrm{i}}(t)$ in both frequency and phase

When both the frequency and the phase of the perturbing force match those of the oscillator, it can be ensured that energy is always transferred from the perturbing force to the system, and not the opposite. Otherwise, the phase would drift in time and the energy would not increase monotonically in time, but would instead oscillate more or less randomly. In a laboratory experiment, a convenient way to deliver perturbations with resonant frequency and phase match is to use a PLL circuit.

A PLL is a closed-loop feedback system that generates and outputs a signal in response to the frequency and phase of an input ("reference") signal. It consists of a phase detector (PD), a loop filter, and a voltage-controlled oscillator (VCO), as shown in Fig. 4. There can also be a frequency divider in the feedback path or in the reference path, or both, in order to make the PLL's output clock a rational multiple of the reference signal. The phase detector generates an output voltage $V_{\mathrm{pd}}(t)$ proportional to the phase error $\theta_{e}$ between the input signal $V_{\mathrm{i}}(t)$ and the output $V_{\mathrm{o}}(t)$ of the VCO: $V_{\mathrm{pd}}(t)=K_{\mathrm{pd}} \theta_{e}$, where $K_{\mathrm{pd}}$ is the PD gain. The alternating current components of the output of the PD are filtered out by the loop filter. The output of the VCO has a frequency that depends on the voltage $V_{\mathrm{f}}(t)$ input to it and is given by $\omega_{2}(t)=$ $\omega_{0}+K_{\mathrm{V}} V_{\mathrm{f}}(t)$, where $\omega_{0}$ is the center frequency of the VCO and $K_{\mathrm{V}}$ is the VCO gain. The PLL works by matching the output frequency $\omega_{2}$ of the VCO with the input frequency $\omega_{1}$. The PD compares the two input frequencies and produces a signal proportional to the phase difference between them. If the frequencies $\omega_{1}$ and $\omega_{2}$ do not match, the PD produces a phase-error signal, which causes the output frequency $\omega_{2}$ of the VCO to change toward the input frequency $\omega_{1}$.

Some characterizing quantities of a PLL are as follows. When there is no input, the VCO oscillates at a frequency $\omega_{0}$ known as the free running frequency of the PLL. The center frequency can be adjusted to any desired value by small design changes, which allows us to operate the PLL at a suitable initial frequency. The capture range $\Delta \omega_{\mathrm{C}}$ of a PLL is the frequency range over which it can initially acquire a lock. Once a lock has been acquired, the PLL continues to track the input frequency over a range known as the lock range $\Delta \omega_{\mathrm{L}}$. If the input frequency varies beyond the lock range, the locked loop will become unlocked. The capture range is usually not greater than the lock range. An important component of the PLL is the loop filter $G(s)$, which filters out noise present in the input signal and prevents the circuit from locking on to unwanted harmonics in the input. This feature makes it possible to track the frequency and phase of a "target" circuit and drive it with resonant perturbations even in the presence of noise. 
Fig. 5 A schematic diagram of our implementation of PLL using LM565 IC. The frequency of VCO is adjusted by varying $R_{1}$

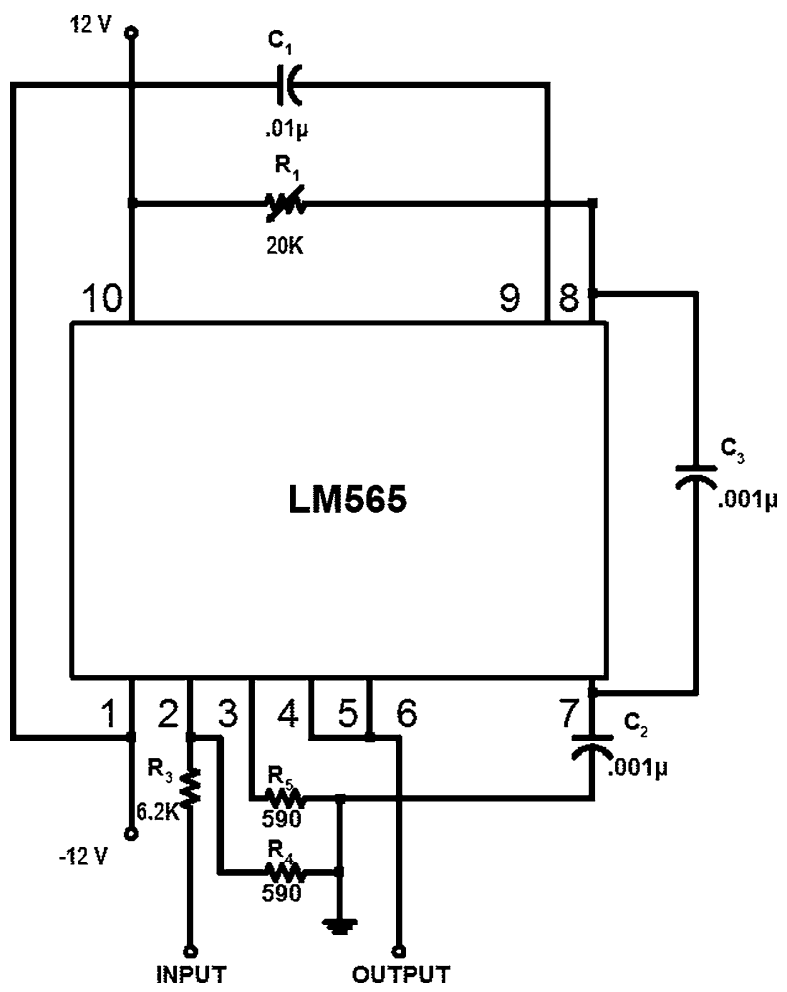

In our experiments, an LM565 PLL integrated circuit is used whose circuitry is shown in Fig. 5. The center frequency is given by $\omega_{0}=0.6 \pi /\left(R_{1} C_{1}\right)$, and the lock range is $\Delta \omega_{\mathrm{L}}= \pm 8 \omega_{0} / V_{\mathrm{cc}}$, where $V_{\mathrm{cc}}$ is the supply voltage to the circuit. The output of the PLL at pins 4 and 5 is a square wave with the same frequency and phase as the input signal at pin 2 . We thus obtain a square wave whose frequency and phase match those of the output of the MOSFET circuit, respectively.

\subsection{Experimental Results}

As shown in Fig. 3, the output $V_{Y}$ of the oscillator is fed to the PLL. A voltage divider at the output of the VCO is used to adjust the amplitude of the perturbing signal. The divider is realized by using a $10 \mathrm{~K} \Omega$ potentiometer. The PLL produces a signal that is matched in phase and frequency with the natural oscillations of the MOSFET oscillator. This signal is then fed back with an appropriate amplitude to the oscillator. The data acquisition system used is the National Instruments PXI Chassis (NI DAQ), which is a complete system with modules for various applications and a built-in computer for storing and processing data. An analog data acquisition module is used in our experiments, since it has the ability to obtain multiple channels of data concurrently with buffer size limited only by the size of the computer hard disk space. Experiments are controlled by a LabView Virtual Instrument (VI) which is programmed for the circuit setup used. The circuit has two measurable outputs: $V_{X}$ and $V_{Y}$. The buffer in the DAQ is configured to collect data in a single shot until a 
Fig. 6 A typical bifurcation diagram of the MOSFET circuit: output $V_{X}$ versus potentiometer resistance $R$

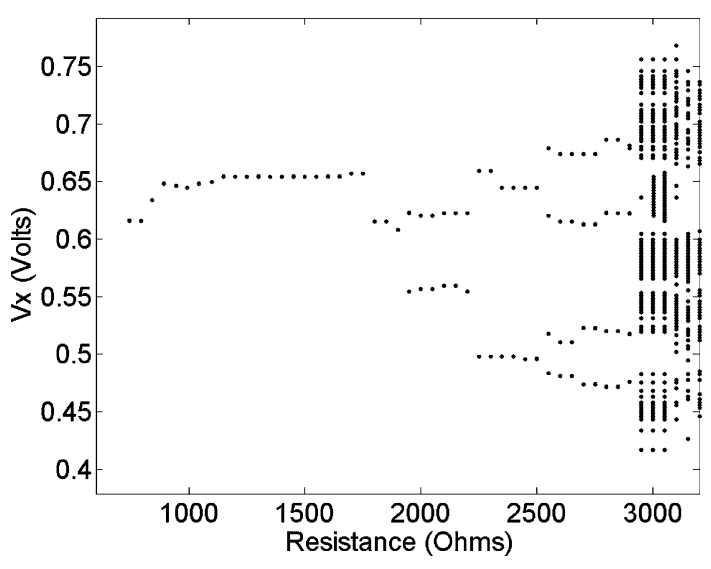

specified number of data points are collected. Data are then unloaded from the buffer in blocks, which are then written to MATLAB readable files. This process ensures that there are no "breaks" in data or overwriting of data in the buffer.

The various states of operation of the oscillator can be observed from a bifurcation diagram that plots a dynamical variable associated with the asymptotic state of the system as a function of some system parameters. By utilizing the standard Poincaré surface-of-section technique, periodic oscillations of period one are represented by a single point in the vertical axis, period-2 oscillations by two points, and so on. Chaotic oscillations are characterized by many (in principle, an infinite number of) points in the diagram. An experimentally obtained bifurcation diagram of the circuit is shown in Fig. 6, where we observe a number of period-doubling bifurcations and a transition to chaos, which are typical features of nonlinear dynamical systems.

An example of inducing chaos is shown in Figs. 7 and 8. When resonant perturbations are absent (i.e., without PLL), the MOSFET oscillator exhibits periodic oscillations of period 2, as shown in the left panel in Fig. 7, where the bifurcation parameter is $R=2.2 \mathrm{k} \Omega$. As soon as the PLL is connected to the oscillator to deliver resonant perturbations, chaos arises, as shown in the left panel in Fig. 8. To quantify the nature of the voltage oscillations in Figs. 7 and 8, we calculate the largest Lyapunov exponent from one of the measured voltage signals [8]. This exponent is the rate of exponential separation of nearby trajectories in time. A positive exponent indicates a sensitive dependence on initial conditions, the hallmark of chaos. Since we assume that the system equations are unknown, it is necessary to reconstruct the phase space based on a measured signal. We use the voltage signal $v_{X}(t)$ and reconstruct from it a seven-dimensional dynamical system using the standard delay-coordinate embedding method [17]. A method for estimating the largest Lyapunov exponent is to directly monitor the growth of an infinitesimal vector in the reconstructed phase space [19]. In particular, let $\mathbf{x}_{n}$ be a state vector in the reconstructed phase space at time $n$ and $\mathbf{y}_{n}$ be its nearest-neighboring vector. Given a long time series $Y_{1}(t)$ and sampling time interval $t_{\mathrm{s}}$, we assume that we have $N_{0}$ iterations available for the phase-space vectors $\mathbf{x}_{n}$ and $\mathbf{y}_{n}$, where one iteration corresponds to one sampling interval. After $k$ iterations, the distance between the two vectors becomes $\left|\mathbf{y}_{n+k}-\mathbf{x}_{n+k}\right|$. We can then 

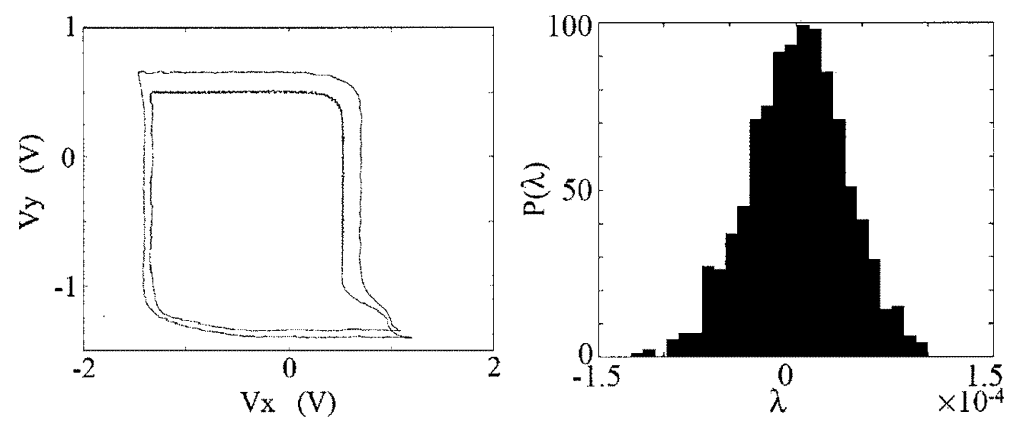

Fig. 7 In the absence of resonant perturbations, a period-2 attractor in the MOSFET oscillator. Left panel: attractor in the phase space. Right panel: histogram of the largest finite-time Lyapunov exponent, which is centered about zero
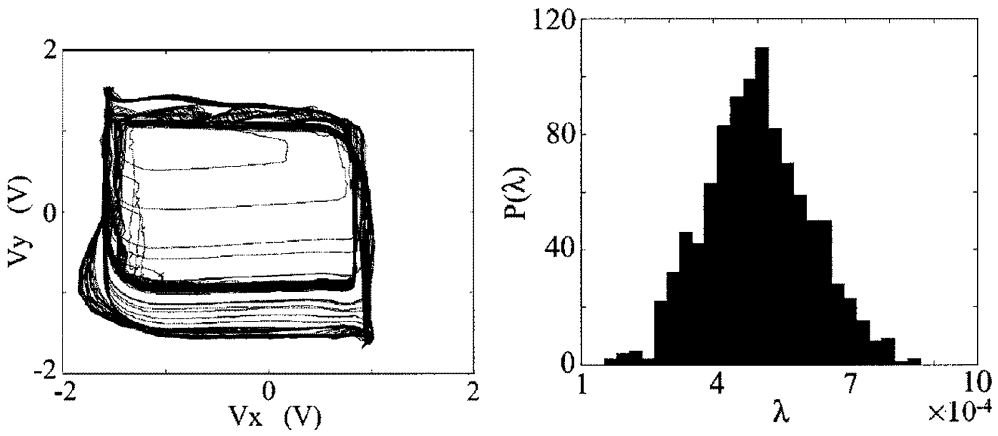

Fig. 8 In the presence of resonant perturbations, a chaotic attractor in the MOSFET oscillator. Left panel: attractor in the phase space. Right panel: histogram of the largest finite-time Lyapunov exponent. The center of the histogram apparently assumes a positive value, indicating chaos

define the prediction error as

$$
p(k)=\frac{1}{N t_{\mathrm{s}}} \sum_{n=1}^{N} \log _{2} \frac{\left|\mathbf{y}_{n+k}-\mathbf{x}_{n+k}\right|}{\left|\mathbf{y}_{n}-\mathbf{x}_{n}\right|},
$$

where $N_{0}$ iterations are used to search for the nearest neighbors, $N<N_{0}$, and $k<N_{0}$. The slope of $p(k)$ is an estimate of the largest Lyapunov exponent.

In our experiments, since the exponent is calculated using a time series of finite length, it exhibits large fluctuations. A meaningful method is then to calculate a statistical distribution (histogram) of the exponents obtained from an ensemble of voltage signals. The right panel of Fig. 7 shows a histogram of the exponent for the period-2 attractor. The histogram centers about zero, indicating that the largest Lyapunov exponent is zero, as it should be for a periodic attractor. When chaos has been induced, the exponent becomes positive, as shown in the right panel of Fig. 8. 


\section{Conclusions}

We have constructed a nonlinear oscillator based on MOSFETs and have demonstrated that chaos can be induced by the method of resonant perturbations. The two essential ingredients of the perturbations are frequency and phase match so that the perturbing signal feeds energy into the oscillator persistently to induce and enhance instability, eventually leading to chaos. We have argued that PLLs are naturally suitable for generating the required resonant perturbations. The loop filter in the PLL circuit ensures accurate tracking of the frequency and phase of the input signal even in a noisy environment. The PLL-based scheme of inducing chaos is thus robust. Inducing and maintaining chaos can find applications, for example, in communications [18] and biomedical engineering. We expect our work to be potentially useful in situations where robust chaos is required or desired.

Acknowledgement This work was supported by AFOSR under Grant No. FA9550-06-1-0024.

\section{References}

1. S.M. Booker, P.D. Smith, P.V. Brennan, R.J. Bullock, Designing input signals to disrupt commercial systems in band-a nonlinear dynamics approach. IEEE Trans. Circuits Syst. I 49, 639-645 (2002)

2. Y. Braiman, I. Goldhirsch, Taming chaotic dynamics with weak periodic perturbations. Phys. Rev. Lett. 66, 2545-2548 (1991)

3. R. Chacón, Maintenance and suppression of chaos by weak harmonic perturbations: a unified view. Phys. Rev. Lett. 86, 1737-1740 (2001)

4. L. Fronzoni, M. Giocondo, M. Pettini, Experimental evidence of suppression of chaos by resonant parametric perturbations. Phys. Rev. A 43, 6483-6487 (1991)

5. J. Guckenheimer, P. Holmes, Nonlinear Oscillations, Dynamical Systems, and Bifurcations of Vector Fields (Springer, New York, 1983)

6. V. In, S.E. Mahan, W.L. Ditto, M.L. Spano, Experimental maintenance of chaos. Phys. Rev. Lett. 74, 4420-4423 (1995)

7. V. In, M.L. Spano, M. Ding, Maintaining chaos in high dimensions. Phys. Rev. Lett 80, 700-703 (1998)

8. A. Kandangath, S. Krishnamoorthy, Y.-C. Lai, J.A. Gaudet, Inducing chaos in electronic circuits by resonant perturbations. IEEE Trans. Circuits Syst. I 54, 1109-1119 (2007)

9. Y.C. Lai, C. Grebogi, Converting transient chaos into sustained chaos by feedback-control. Phys. Rev. E 49, 1094-1098 (1994)

10. Y.-C. Lai, A. Kandangath, S. Krishnamoorthy, J.A. Gaudet, A.P.S. de Moura, Inducing chaos by resonant perturbations: theory and experiment. Phys. Rev. Lett. 94, 214101 (2005)

11. R. Lima, M. Pettini, Suppression of chaos by resonant parametric perturbations. Phys. Rev. A 41, 726-733 (1990)

12. Y. Nagai, Y.C. Lai, Selection of a desirable chaotic phase using small feedback-control. Phys. Rev. E 51, 3842-3848 (1995)

13. Z. Qu, G. Hu, G. Yang, G. Qin, Phase effect in taming nonautonomous chaos by weak harmonic perturbations. Phys. Rev. Lett. 74, 1736-1739 (1995)

14. A.G. Radwan, A.M. Soliman, A.-L. El-Sedeek, MOS realization of the double scroll-like chaotic equation. IEEE Trans. Circuits Syst. I 50, 285-288 (2003)

15. S.J. Schiff, K. Jerger, D.H. Duong, T. Chang, M.L. Spano, W.L. Ditto, Controlling chaos in the brain. Nature 370, 615-620 (1994)

16. I.B. Schwartz, I. Triandaf, Sustaining chaos by using basin boundary saddles. Phys. Rev. Lett. 77, 4740-4743 (1996)

17. F. Takens, Detecting strange attractors in fluid turbulence, in Dynamical Systems and Turbulence, ed. by D. Rand, L.S. Young. Lecture Notes in Mathematics (Springer, Berlin, 1981), pp. 366-381 
18. W.M. Tam, F.C.M. Lau, C.K. Tse, Generalized correlation-delay-shift-keying scheme for noncoherent chaos-based communication systems. IEEE Trans. Circuits Syst. I 53, 712-721 (2006)

19. A. Wolf, J.B. Swift, L. Swinney, J.A. Vastano, Determining Lyapunov exponents from a time series. Physica D 16, 285-317 (1985)

20. W.M. Yang, M.Z. Ding, A.J. Mandell, E. Ott, Preserving chaos: control strategies to preserve complex dynamics with potential relevance to biological disorders. Phys. Rev. E 51, 102-110 (1995)

21. A.M. Young, C.P. Silva, Nonlinear communications engineering: high frequency forced duffing oscillator circuit. Aerospace Corporation Technical Report, June 2002 